\title{
STAT3 enhances radiation-induced tumor migration, invasion and stem-like properties of bladder cancer
}

\author{
FANG WANG ${ }^{*}$, XIANGLI MA* , GUANGMIN MAO, XIANGYAN ZHANG and ZHAOLU KONG \\ Department of Radiobiology, Institute of Radiation Medicine, Fudan University, Shanghai 200032, P.R. China
}

Received May 1, 2020; Accepted October 21, 2020

DOI: $10.3892 / \mathrm{mmr} .2020 .11728$

\begin{abstract}
Bladder cancer (BCa) is the most common cancer of the human urinary system, and is associated with poor patient prognosis and a high recurrence rate. Cancer stem cells (CSCs) are the primary cause of tumor recurrence and metastasis, possessing self-renewal properties and resistance to radiation therapy. Our previous studies indicated that phosphorylated signal transduction and transcription activator 3 (STAT3) may be a potential biomarker to predict radiation tolerance and tumor recurrence in patients with $\mathrm{BCa}$, following conventional radiotherapy. The aim of the present study was to investigate the underlying mechanism of STAT3 in the radio-resistance of $\mathrm{BCa}$ cells. It was found that fractionated irradiation promoted the activation of two STAT3-associated CSCs signaling pathways in $\mathrm{BCa}$ cells, namely suppressor of variegation 3-9 homolog 1/GATA binding protein 3/STAT3 and Janus kinase 2/STAT3. Surviving cells exhibited elevated migratory and invasive abilities, enhanced CSC-like characteristics and radio-resistance. Furthermore, knockdown of STAT3 expression or inhibition of STAT3 activation markedly decreased the self-renewal ability and tumorigenicity of radiation-resistant BCa cells. Kaplan-Meier analysis revealed that decreased STAT3 mRNA levels were associated with increased overall survival times in patients with BCa. Taken together, these data indicated that STAT3 may be an effective therapeutic target for inhibiting the progression, metastasis and recurrence of $\mathrm{BCa}$ in patients receiving radiotherapy.
\end{abstract}

\section{Introduction}

Bladder cancer $(\mathrm{BCa})$ is a heterogeneous disease that commonly presents as a malignant tumor; there are two main subtypes,

Correspondence to: Dr Zhaolu Kong, Department of Radiobiology, Institute of Radiation Medicine, Fudan University, 2094 Xietu Road, Shanghai 200032, P.R. China

E-mail: kongzhaolu@fudan.edu.cn

*Contributed equally

Key words: signal transduction and transcription activator 3, radiosensitivity, cancer stem cell, bladder cancer non-muscle invasive (NMIBC) and muscle invasive (MIBC) $\mathrm{BCa}$ (1). Although $70 \%$ of patients are diagnosed with NMIBC, the disease can quickly progress to invade the muscle layer (2). The current treatment for NMIBC consists of transurethral resection of the bladder tumor in conjunction with intravesical chemotherapy or immunotherapy (3). MIBC accounts for $30 \%$ of all BCa cases at initial presentation, and is associated with a higher death rate due to distant metastasis (1). Even after receiving aggressive radical cystectomy (RC), the 5-year mortality rate of patients with MIBC remains at 50-70\%, with a high recurrence rate and poor overall prognosis (3).

For decades, RC has been the primary treatment method for MIBC; however, improved survival rates cannot be achieved through surgery alone (4). In the past 20 years, as MIBC treatments have aimed to preserve the bladder and improve quality of life, radiation therapy (RT), chemotherapy for radiation sensitization, and immunotherapy have continuously improved (5). This has increased the 5-year overall survival (OS) rate to 40-50\%, resulting in improved patient quality of life (6). For patients that are medically unsuitable for RC, or those who prefer non-surgical alternatives, RT and concurrent chemotherapy are currently the most effective treatments. For suitable surgical candidates, bladder conservation can maintain function and result in similar oncologic outcomes to RC (7). However, the resistance of cancer cells to radiation often limits the effectiveness of RT. Furthermore, patients may still experience local tumor recurrence, and acquiring radio-resistance (RR) after initial radiotherapy may exacerbate local tumor recurrence and metastasis $(8,9)$. At present, the mechanisms by which cancer cells acquire RR remain unclear. In the present study, obstacles in the treatment of $\mathrm{BCa}$ were considered to be attributed to the radiation tolerance of cancer stem cells (CSCs) present in the tumor $(10,11)$. CSCs may be seen as a reservoir of cancer cells due to their self-renewal and plasticity properties, and the ability to reconstruct heterogeneous tumor cell populations (12). Although there are a lack of supporting clinical data, experimental data and preliminary clinical trials suggest that CSC-targeted treatment has the potential to improve radiotherapeutic efficacy $(13,14)$.

Signal transduction and transcription activator 3 (STAT3) is a transcription factor with a number of important biological functions. Increasing evidence suggests that STAT3 is an important regulator of normal and cancer stem cells (15). It is involved in epithelial-to-mesenchymal 
transition (EMT)-associated pathways, which are hypothesized to be the primary mechanisms for CSC generation (16), and has the ability to promote cancer progression by regulating the activity of CSCs (17). Moreover, our previous studies revealed that high levels of STAT3 phosphorylation are closely associated with the acquired radiation resistance (ARR) of urinary system tumors $(18,19)$. The present study aimed to elucidate the role of STAT3 in RR and its association with the CSC phenotype in BCa cells. The data suggested that the aberrant activation of STAT3 enhanced the migration, invasion and stem-like properties of $\mathrm{BCa}$ cells in response to long-term ionizing radiation (IR) exposure.

\section{Materials and methods}

Cell culture and treatment. Human BCa cell lines (5637 and T24) were purchased from The Cell Bank of Type Culture Collection of the Chinese Academy of Sciences and cultured in high glucose Dulbecco's modified Eagle's medium (DMEM; Invitrogen; Thermo Fisher Scientific, Inc.) containing $10 \%$ fetal bovine serum (FBS; Invitrogen; Thermo Fisher Scientific, Inc.), $100 \mathrm{U} / \mathrm{ml}$ penicillin and $100 \mathrm{U} / \mathrm{ml}$ streptomycin (Sigma-Aldrich; Merck KGaA). The cells were maintained at $37^{\circ} \mathrm{C}$ in a humidified atmosphere with $5 \% \mathrm{CO}_{2}$. Cells were irradiated at room temperature in ambient air using a ${ }^{137} \mathrm{Cs}$ source ( $\gamma$-ray; Nordion, Inc.) at a dose rate of $0.79 \mathrm{~Gy} / \mathrm{min}$. Resistant cells were generated by mimicking clinical radiotherapy treatment as previously described (19). Subsequently, cells that survived irradiation with 60 Gy (2 Gy per day, 5 days per week, and continuous exposure until the cumulative dose reached 60 Gy) were defined as 5637R and T24R cells.

Colony formation assay. A colony formation assay was used to determine the sensitivity of cells to IR, as previously described (19). Briefly, the cells were irradiated with increasing doses of $\gamma$-rays $4 \mathrm{~h}$ post-IR, and then plated into $60-\mathrm{mm}$ dishes in triplicate. After 14 days of incubation, visible colonies (>50 cells) were stained and counted. The survival fraction curve was plotted using SigmaPlot 11.0 (Systat Software, Inc.).

Cell treatment. The aforementioned cell lines were seeded into $60-\mathrm{mm}$ dishes at $5 \times 105$ cells/dish. At $60-70 \%$ confluency, $50 \mu \mathrm{M}$ STAT3 Inhibitor VI S3I-201 (Santa Cruz Biotechnology, Inc.) was added to the appropriate dishes, and the cells were cultured once more (20). After $24 \mathrm{~h}$, the medium was discarded and the cells were harvested by trypsinization for tumor sphere formation, soft agar colony formation and western blot assays.

SmallinterferingRNA(siRNA)transfection.siSTAT3 andthenegative control siRNA were designed and constructed by Shanghai GenePharma Co., Ltd. The targeting sequence for siSTAT3 was 5'-CAGGCTGGTAATTTATATAAT-3', and that of the negative control siRNA was 5'-CATTGACTTATAAATTCGTTC-3'. Transient STAT3 inhibition was achieved by transfection with

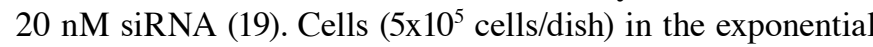
growth phase were seeded into 60-mm dishes, and transfection was performed at $70 \%$ confluency. The cells were transfected using Lipofectamine ${ }^{\circledR} 2000$ reagent (Thermo Fisher Scientific, Inc.) in DMEM, according to the manufacturer's instructions.
After $24 \mathrm{~h}$, the medium was changed and subsequent experiments were performed.

Western blot analysis. The expression levels of STAT3, phosphorylated STAT3 (pSTAT3 ${ }^{\text {Ser727 }}$ and pSTAT3 ${ }^{\text {Tyr705}}$ ), matrix metalloproteinase (MMP)-2, MMP-9, suppressor of variegation 3-9 homolog 1 (KMT1A), GATA binding protein 3 (GATA3) and Janus kinase 2 (JAK2), epidermal growth factor receptor (EGFR), phosphorylated EGFR (p-EGFR) were assessed using total cellular protein. The cellular proteins were extracted using M-PER ${ }^{\mathrm{TM}}$ Mammalian Protein Extraction Reagent (Thermo Fisher Scientific, Inc.) and quantified using a BCA Protein Assay Kit (Thermo Fisher Scientific, Inc.), where the ratio of protease inhibitor $\left(\right.$ Halt $^{\mathrm{TM}}$ Protease Inhibitor Single-Use Cocktail; Thermo Fisher Scientific, Inc.) to protein lysate was 1:100. Equal amounts of each sample (40 $\mu \mathrm{g})$ were fractionated using 8 and 12\% SDS-PAGE gels, followed by transfer to $0.45-\mu \mathrm{m}$ PVDF membranes; the fractionation and transfer times were controlled according to the molecular weights of the proteins. The membrane was washed with TBS with $0.05 \%$ Tween-20 solution and blocked with 5\% BSA (Beyotime Institute of Biotechnology) at room temperature for $1 \mathrm{~h}$. The membranes were subsequently probed with primary antibodies against pSTAT3 ${ }^{\text {Ser727 }}$ (cat. no. ET1607-39; 1:1,000; Hangzhou HuaAn Biotechnology Co., Ltd.), pSTAT3 ${ }^{\mathrm{Ty} 705}$ (cat. no. ET1603-40; 1:1,000; Hangzhou HuaAn Biotechnology Co., Ltd.), STAT3 (cat. no. ET1607-38; 1:1,000; Hangzhou HuaAn Biotechnology Co., Ltd.), MMP-2 (cat. no. sc-13594; 1:1,000; Santa Cruz Biotechnology, Inc.), MMP-9 (cat. no. sc-393859; 1:1,000; Santa Cruz Biotechnology, Inc.), KMT1A (cat. no. sc-377112; 1:1,000; Santa Cruz Biotechnology, Inc.), GATA3 (cat. no. sc-269; 1:1,000; Santa Cruz Biotechnology, Inc.), JAK2 (cat. no. sc-390539; 1:1,000; Santa Cruz Biotechnology, Inc.), EGFR (cat. no. sc-373746; 1:100; Santa Cruz Biotechnology, Inc.) and p-EGFR (cat. no. sc-377547; 1:100; Santa Cruz Biotechnology, Inc.) overnight at $4^{\circ} \mathrm{C}$. GAPDH (cat. no. AB-P-R001; 1:1,000; Hangzhou Goodhere Biotechnology Co., Ltd.) was used as the internal loading control. The membranes were then incubated with anti-mouse (cat. no. HA1006; 1:1,000) or anti-rabbit (cat. no. HA1001; 1:1,000) horseradish peroxidase-conjugated secondary antibodies (both from Hangzhou HuaAn Biotechnology Co., Ltd.) for $1 \mathrm{~h}$ at room temperature. Finally, the proteins were visualized using the ChemiDoc $^{\mathrm{TM}}$ XRS System (Bio-Rad Laboratories, Inc.).

Tumor sphere formation. A total of $5 \times 10^{3} \mathrm{BCa}$ cells were seeded into ultra-low attachment surface 6-well plates (Corning, Inc.) and maintained in DMEM with $20 \mathrm{ng} / \mathrm{ml}$ epidermal growth factor (PeproTech, Inc.), $20 \mathrm{ng} / \mathrm{ml}$ basic fibroblast growth factor (PeproTech, Inc.) and 2\% B27 (Thermo Fisher Scientific, Inc.). After 1 week of cultivation, the number of tumor spheres was counted in five independent fields (21). The experiments were repeated three times.

Cell migration assay. 5637, 5637R, T24 and T24R cell migration were determined using $8.0-\mu \mathrm{m}$ pore size Transwell inserts (BD Biosciences) placed in 24-well plates. For the 5637 and 5637R lines, $2 \times 10^{5}$ cells in $500 \mu$ l FBS-free DMEM were seeded into the upper chambers; for the T24 and T24R, $1 \times 10^{4}$ cells were used. DMEM containing $10 \%$ FBS $(1,300 \mu \mathrm{l})$ 
was added to the lower chambers. Following incubation for $24 \mathrm{~h}$ at $37^{\circ} \mathrm{C}$, the upper chambers were carefully removed and washed three times with phosphate-buffered saline (PBS; $\mathrm{pH}$ 7.4). The cells were fixed in $100 \%$ methyl alcohol (Sangon Biotech Co., Ltd.) at room temperature for $15 \mathrm{~min}$, and the chambers were stained with $0.2 \%$ crystal violet (Sangon Biotech Co., Ltd.) for 15-30 min at room temperature. When the chambers were dry, the cells were analyzed under a light microscope (DFC450-C; Leica Microsystems GmbH) at x100 magnification. The experiments were repeated three times.

Cell invasion assay. Transwell chambers (Corning, Inc.) precoated with Matrigel (BD Biosciences) for $2 \mathrm{~h}$ at $37^{\circ} \mathrm{C}$ were used to assess cell invasion capacity. The invasiveness of 5637 , 5637R, T24 and T24R cells was determined using $8.0-\mu \mathrm{m}$ pore size Transwell inserts (BD Biosciences) in 24-well plates. A total of $2 \times 10^{5} 5637$ and $5637 \mathrm{R}$ cells were seeded into the upper chambers with $400 \mu \mathrm{l}$ FBS-free DMEM, and $1 \times 10^{4}$ T24 and T24R cells were used in the same manner; 1,300 $\mu \mathrm{l}$ DMEM containing $10 \%$ FBS was added to the lower chambers. Following incubation for $29 \mathrm{~h}$ at $37^{\circ} \mathrm{C}$, the upper chambers were removed and washed with $\mathrm{PBS}$ as aforementioned. The cells were then fixed in $100 \%$ methyl alcohol for $15 \mathrm{~min}$, and the chambers were stained with $0.2 \%$ crystal violet for 15-30 min, both at room temperature. After drying, the cells were analyzed using a light microscope (magnification, x100), and the experiments were repeated three times.

Soft agar colony formation. Low-melting agarose solution (Sigma-Aldrich; Merck KGaA) was prepared using ultrapure water (concentration, 1.2 and 0.8\%). Sterile 2X DMEM, (2\% 2xPS and 20\% FBS) was prepared at room temperature. For $1.2 \%$ agarose, $2 \mathrm{X}$ DMEM was added to the agarose solution at a $1: 1$ ratio; $3 \mathrm{ml}$ of the mixture was then added to $6-\mathrm{cm}$ dishes, which was cooled and solidified in an incubator. For $0.8 \%$ agarose, $2 \mathrm{X}$ DMEM was mixed with the agar solution (1:1 ratio), and a single cell suspension was added. The solution was thoroughly mixed and layered on top of the $1.2 \%$ agar in the 6-cm dishes. After the upper layer of agarose had fully solidified, the dishes were incubated at $37^{\circ} \mathrm{C}$ for $12-14$ days. The cells were then stained with $0.2 \%$ crystal violet (Sangon Biotech Co., Ltd.) for 15-30 min at room temperature, and images were captured using a digital camera. The experiments were repeated three times.

Animal model and experimental protocol. A total of 21 male BALB/c nude mice (age, 35-42 days; weight, 22-25 g) were purchased from Shanghai SLAC Laboratory Animal Co., Ltd. Experimental animals were housed at $24^{\circ} \mathrm{C}, 50-70 \%$ humidity, with a 12-h light/dark cycle and free access to food and water. All experiments were approved by the Committee for Ethical Use of Experimental Animals at Fudan University (approval no. 201802144S; Shanghai, China). For the generation of xenografts, $1 \times 10^{6} \mathrm{BCa}$ cells (in $0.1 \mathrm{ml} \mathrm{PBS}$ ) were subcutaneously injected into the back of each mouse. The first batch of mice were randomly divided into the following five groups (n=3/group): i) Saline control group; ii) 5637 group; iii) $5637 \mathrm{R}$ group; iv) T24 group; and v) T24R group. The second batch were randomly divided into the T24 si control ( Ctrl) and T24 siSTAT3 groups ( $n=3 /$ group). After 7 days, the xenograft volumes were measured every 2 days. Tumor volume $\left(\mathrm{mm}^{3}\right)$ was measured by caliper and calculated using the following equation: $\left.\mathrm{V}=\left(\mathrm{L}_{\mathrm{x} \mathrm{W}}\right)^{2}\right) / 2$, where $\mathrm{V}$ is the tumor volume, $\mathrm{L}$ is the length and $\mathrm{W}$ is the width of the tumor. On day 30 , all mice were placed into the $4 \%$ isoflurane chamber until animals lost consciousness, then cervical dislocation was performed to euthanize the mice. No toe reflex of muscle tone should be present at this point. The tumors were dissected immediately and fixed with $10 \%$ formalin overnight at $4^{\circ} \mathrm{C}$.

Kaplan-Meier Plotter database analysis. The survival and gene expression information of 405 patients with BCa was obtained from the Kaplan-Meier Plotter online database, (https://kmplot. com/analysis/), and the association between STAT3 mRNA expression and clinical prognosis was evaluated. All clinical datasets were acquired from published literature, and written informed consent was previously obtained (22). Specifically, patient samples were split into two groups according to the median expression of STAT3 mRNA in the tumor tissue: STAT $3^{\text {high }}$ vs. STAT3 ${ }^{\text {low }}$ (computer optimization). By setting the threshold as 'all', patient OS and relapse-free survival (RFS) times were plotted using the Kaplan-Meier survival assay. The 95\% confidence interval (CI), hazard ratio (HR) and log rank P-value were subsequently calculated.

Reverse transcription-quantitative PCR (RT-qPCR) analysis. Total RNA was extracted from four types of BCa cell sublines (5637, 5637R, T24, T24R) using TRIzol $^{\circledR}$ reagent (Tiangen Biotech Co., Ltd.), according to the manufacturer's instructions. cDNA was synthesized using the ReverTra Ace ${ }^{\mathrm{TM}}$ qPCR RT Kit (cat. no. FSQ-101; Toyobo Life Science) with the following conditions: $37^{\circ} \mathrm{C}$ for $15 \mathrm{~min}, 98^{\circ} \mathrm{C}$ for $5 \mathrm{~min}$ and stored at $4^{\circ} \mathrm{C}$. Primers for amplification of EGFR and GAPDH were designed by GenScript. Primer sequences were as follows: EGFR forward, 5'-TCCCTCAGCCACCCATATGTAC-3' and reverse, 5'-GTCTCGGGCCATTTTGGAGAATCC-3'; GAPDH forward, 5'-CACCAACTGGGACGACAT-3' and reverse, 5'-ACAGCCTGGATAGCAACG-3'. RT-qPCR amplification reactions were performed using BioEasy Master Mix (SYBR-Green) (cat. no. BSB30L1; Hangzhou Bioer Co., Ltd.) with a Mx3000P Quantitative PCR system (Agilent Technologies, Inc.). Amplification conditions were as follows: $95^{\circ} \mathrm{C}$ for $5 \mathrm{~min}$, followed by 40 cycles of $95^{\circ} \mathrm{C}$ for $30 \mathrm{sec}$ and $60^{\circ} \mathrm{C}$ for $45 \mathrm{sec}$. Each sample was examined in triplicate and the amount of product was normalized relative to that of GAPDH. Quantitative values were calculated according to the $2^{-\Delta \Delta \mathrm{Cq}}$ method (23).

Statistical analysis. All data are presented as the mean \pm SEM, and all experiments were repeated at least three times. Statistical analysis was conducted using GraphPad Prism software (version 8.0; GraphPad Software, Inc.). Student's two-tailed t-test and the log-rank test were used for the determination of statistical relevance between groups. $\mathrm{P}<0.05$ was considered to indicate a statistically significant difference.

\section{Results}

Fractionated irradiation (FI) enhances the motility of human BCa cells. The human BCa cell lines, 5637 and T24, were subjected to 30 FI treatments of 2 Gy/day; surviving cells 

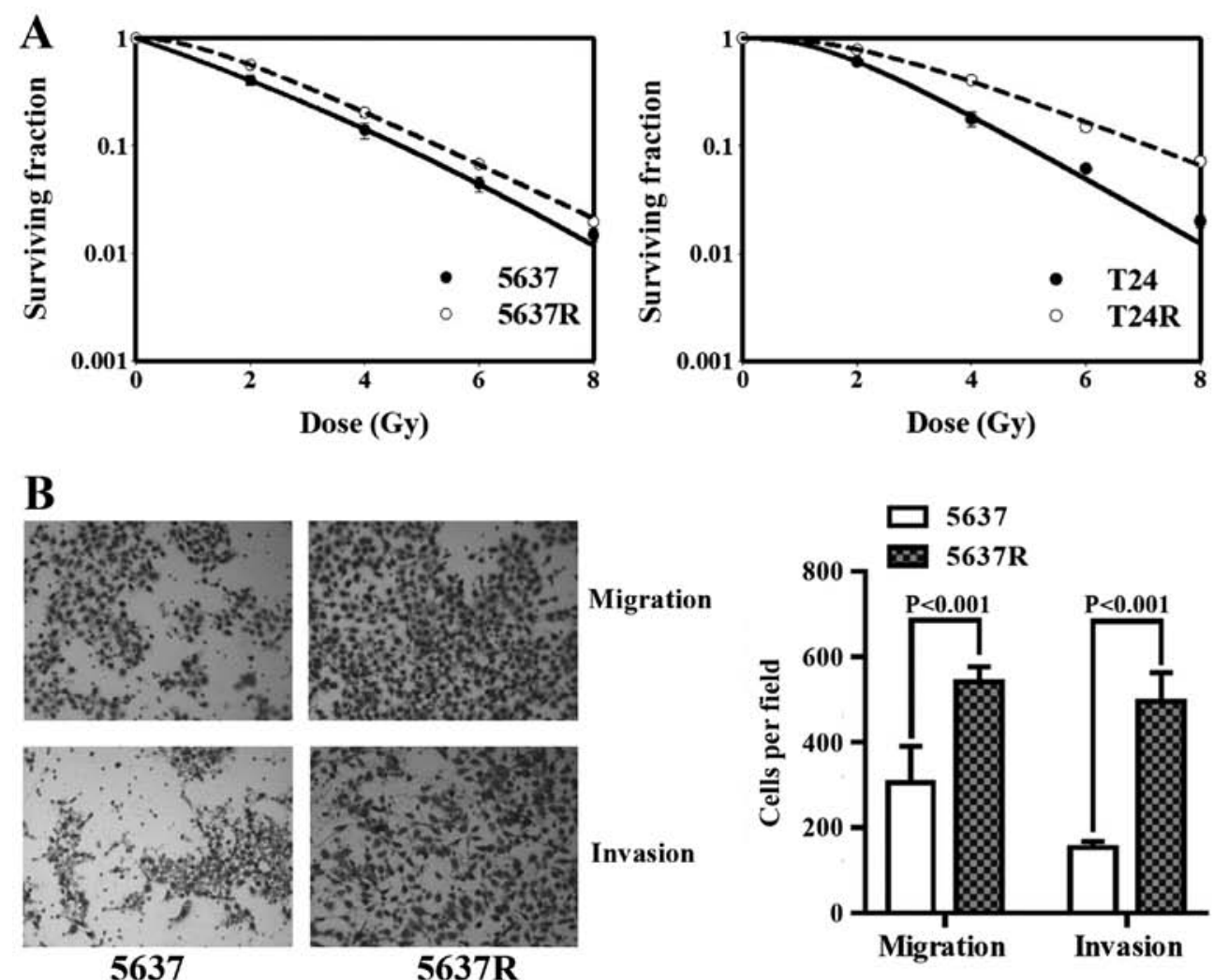

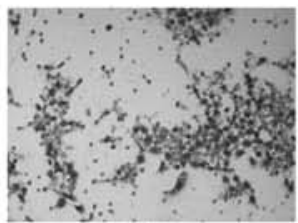

5637
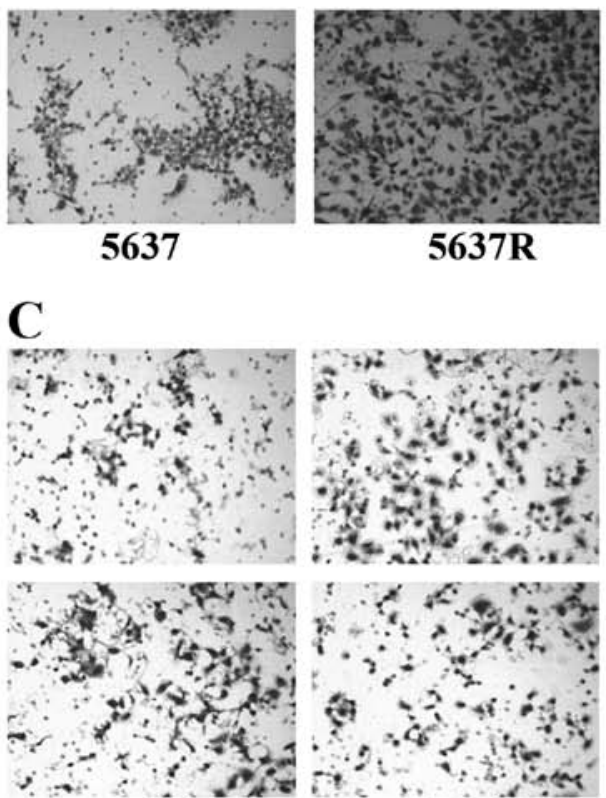

T24
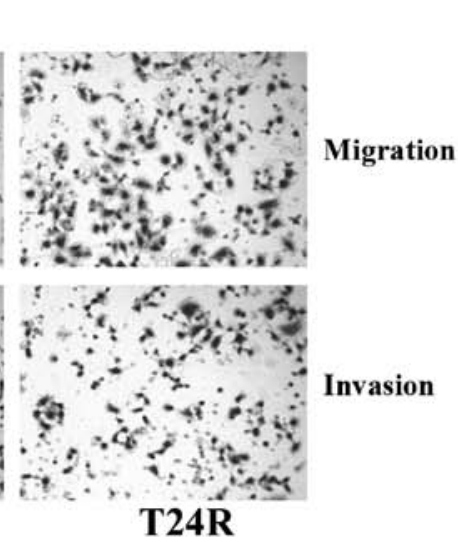

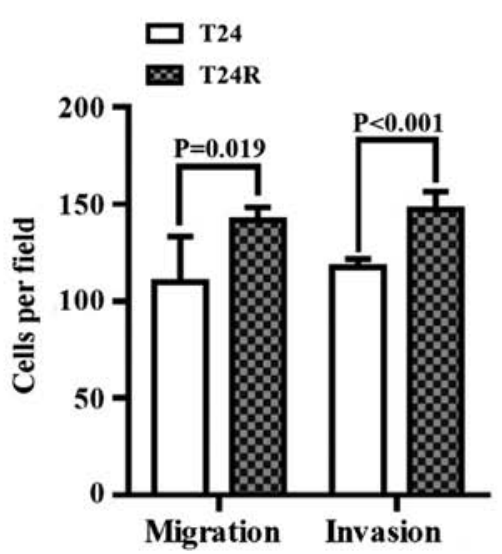

Figure 1. Fractionated irradiation enhances cell motility in human bladder cancer cells. (A) Radio-sensitivity of 5637/5637R and T24/T24R cells was detected via a colony formation assay. (B) Representative images and quantification of migration and invasion analysis of 5637 and $5637 \mathrm{R}$ cells. (C) Representative images and quantification of migration and invasion analysis of T24 and T24R cells. The data are presented as the mean \pm SEM of triplicate experiments. $\mathrm{R}$, radio-resistant cells.

were named 5637R and T24R, respectively. Initially, the radio-tolerance of the surviving cells was compared with that of the respective parental cell lines (Fig. 1A). The surviving 2-Gy fractions $\left(\mathrm{SF}_{2}\right)$ in 5637 and $5637 \mathrm{R}$ cells were $0.42 \pm 0.05$ and $0.57 \pm 0.03$, respectively $(\mathrm{P}=0.011)$, and the $\mathrm{SF}_{2}$ of the T24 and T24R cells were $0.61 \pm 0.04$ and $0.78 \pm 0.05$, respectively $(\mathrm{P}=0.010)$. The effects of FI on cell motility were then investigated. Compared with 5637 and T24 cells, 5637R and T24R cells demonstrated notable increases in both migration and invasion. The migration rates of 5637 and $5637 \mathrm{R}$ cells were $305.8 \pm 85.21$ vs. $540.8 \pm 36.41,(\mathrm{P}<0.001$; Fig. $1 \mathrm{~B})$, and those of T24 and T24R cells were 109.8 \pm 23.40 and $141.8 \pm 6.65(\mathrm{P}=0.019$; Fig. 1C), respectively. The invasion rates of 5637 and $5637 \mathrm{R}$ cells were $153.6 \pm 13.89$ vs. $495.0 \pm 68.12$ ( $\mathrm{P}<0.001$; Fig. $1 \mathrm{~B})$, and those of T24 and T24R cells were 117.6 \pm 4.16 and 147.2 \pm 9.28 , respectively $(\mathrm{P}<0.001$; Fig. $1 \mathrm{C})$. It is worth mentioning that, in order to observe the invasiveness of $\mathrm{BCa}$ cells, the end time was set to $29 \mathrm{~h}$ after inoculation, and the end time of migration experiments was $24 \mathrm{~h}$ post-inoculation.

FI enhances the CSC characteristics of human BCa cells. CSCs primarily reside within tumors and are responsible for cancer recurrence. They exhibit stem cell-like characteristics and resistant phenotypes, with self-renewal and abnormal differentiation potential. As such, the existence of CSCs is closely associated with tumorigenesis and drug resistance $(24,25)$. In the present study, 5637R and T24R cells possessed enhanced CSC characteristics, such as three-dimensional tumorsphere-forming ability, as well as anchorage and clonogenic ability in semi-solid culture (Fig. 2A and C). However, 


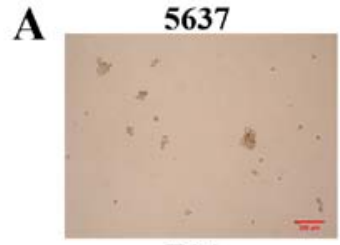

T24

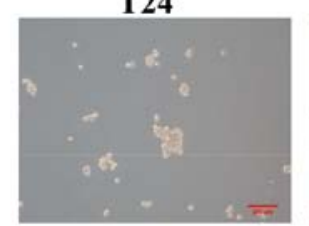

C

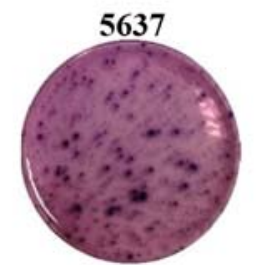

T24

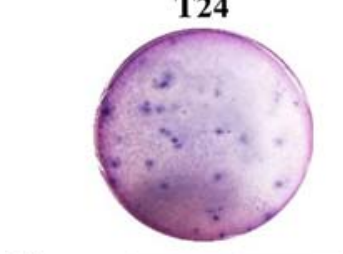

Control

5637

5637R

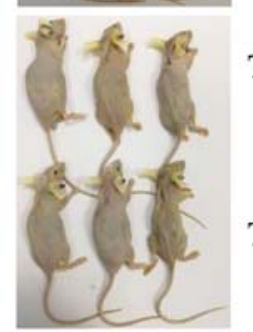

5637R

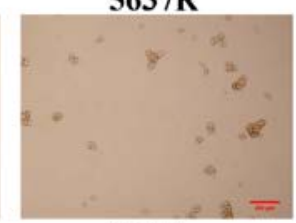

T24R

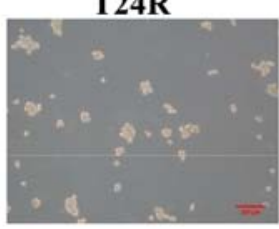

5637R

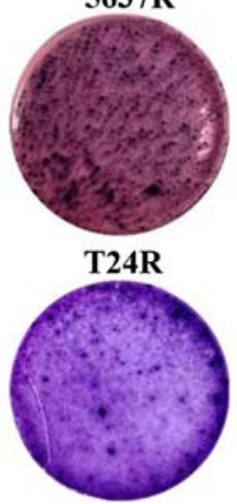

T24

T24R
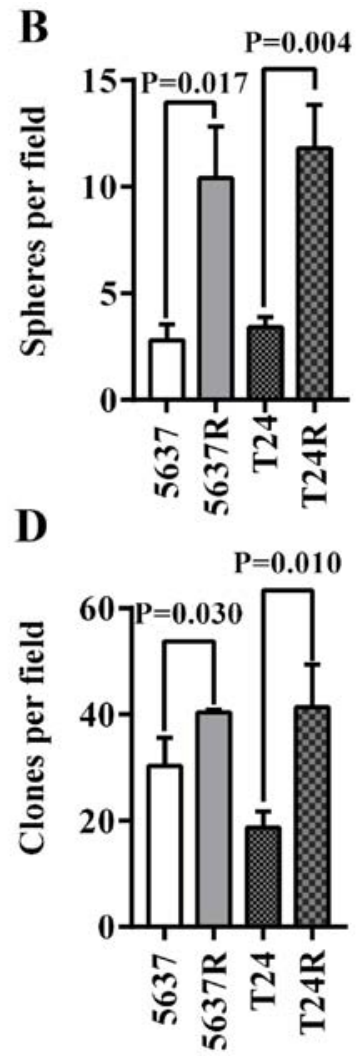

Figure 2. Fractionated irradiation enriches CSC characteristics in human bladder cancer cells. (A) Representative images (scale bar, $200 \mu$ m) and (B) quantification of tumorspheres formed by 5637/5637R and T24/T24R cells. The number of tumorspheres was counted in five independent fields/well after 1 week of cultivation. (C) Representative images and (D) quantification of soft agar colony formation analysis of 5637/5637R and T24/T24R cells. The data are presented as the mean \pm SEM of triplicate experiments. (E) Results of the tumor formation assays of saline control, 5637, 5637R, T24 and T24R groups ( $=3$ ). $\mathrm{R}$, radio-resistant cells; CSC, cancer stem cells.

as there is still controversy concerning the existence of bladder CSCs (BCSCs) (26), 5637R and T24R are referred to as BCSC-like cells in the present study. An increased number of spheres (diameter, $>50 \mu \mathrm{M}$ ) was observed in both $\mathrm{RR}$ cell lines, compared with the parental cells [5637 vs. 5637R, $2.8 \pm 0.75$ vs. $10.4 \pm 2.42(\mathrm{P}=0.017)$; and $\mathrm{T} 24$ vs. T24R, $3.4 \pm 0.49$ vs. $11.8 \pm 2.04(\mathrm{P}=0.004)$; Fig. $2 \mathrm{~B}$ ]. However, there was no notable difference in the size of the tumorspheres formed by the parental BCa cells or their RR counterparts (Fig. 2A). Another common method of identifying stem cells is the low-melting soft agar colony formation assay. Both 5637R and T24R cells formed a greater number of colonies than 5637 and T24 cells [5637 vs. 5637R, $30.3 \pm 5.25$ vs. $40.3 \pm 0.47(\mathrm{P}=0.030)$; and T24 vs. T24R, $18.7 \pm 3.09$ vs. $41.3 \pm 8.06(\mathrm{P}=0.010)$; Fig. $2 \mathrm{D}]$. To further evaluate the potential CSC properties of RR cells, tumor formation capacity was assessed by injecting BCa cells into BALB/c nude mice. Only T24R cells formed tumors in immunodeficient mice and the maximum diameter reached $2 \mathrm{~cm}$ (Fig. 2E).

Elevated STAT3 phosphorylation in RR BCa cells is associated with an increase in tumor cell motility and stemness. To further elucidate the mechanism by which FI promotes BCa recurrence and metastasis, the expression of a series cell movement- and stemness-associated proteins was investigated (Fig. 3A). Consistent with previous reports $(18,19)$, increased levels of pSTAT3 and MMP-2/9 were detected in both RR cell types. Also, the differences in two STAT3-based CSC-related signaling pathways, [KMT1A/GATA3/STAT3 (21) and JAK/STAT3 (27)] were assessed between parental BCa cells and their RR counterparts. As predicted, both signaling pathways were more active in the resistant cells. Expression levels 

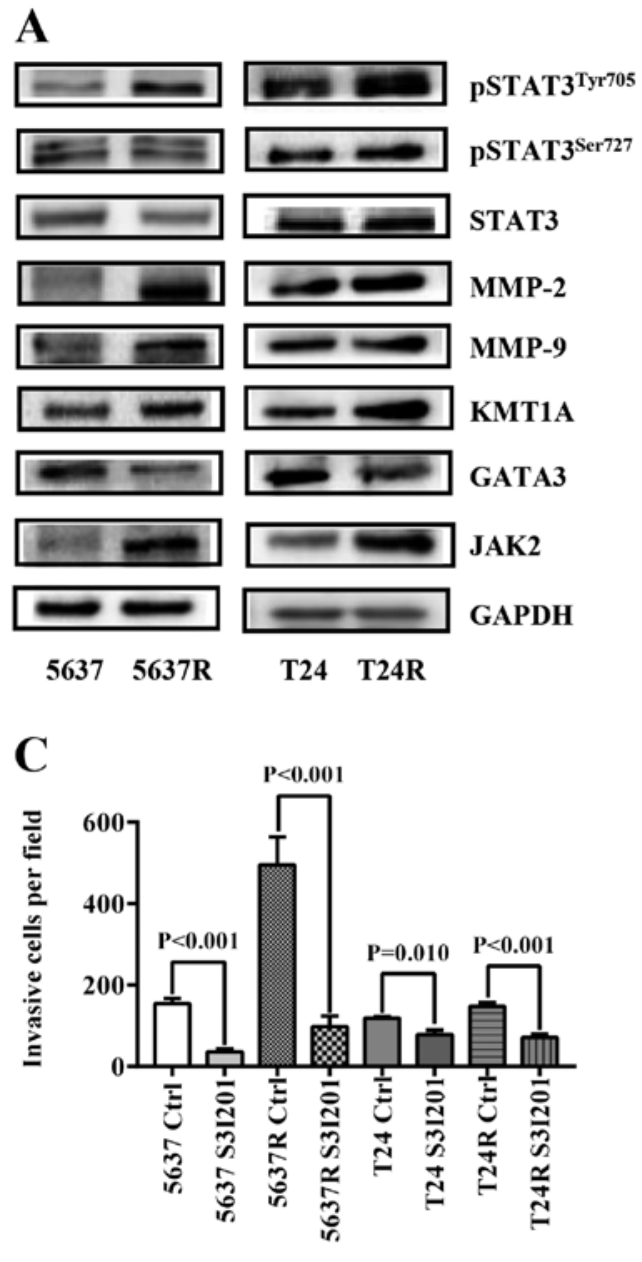
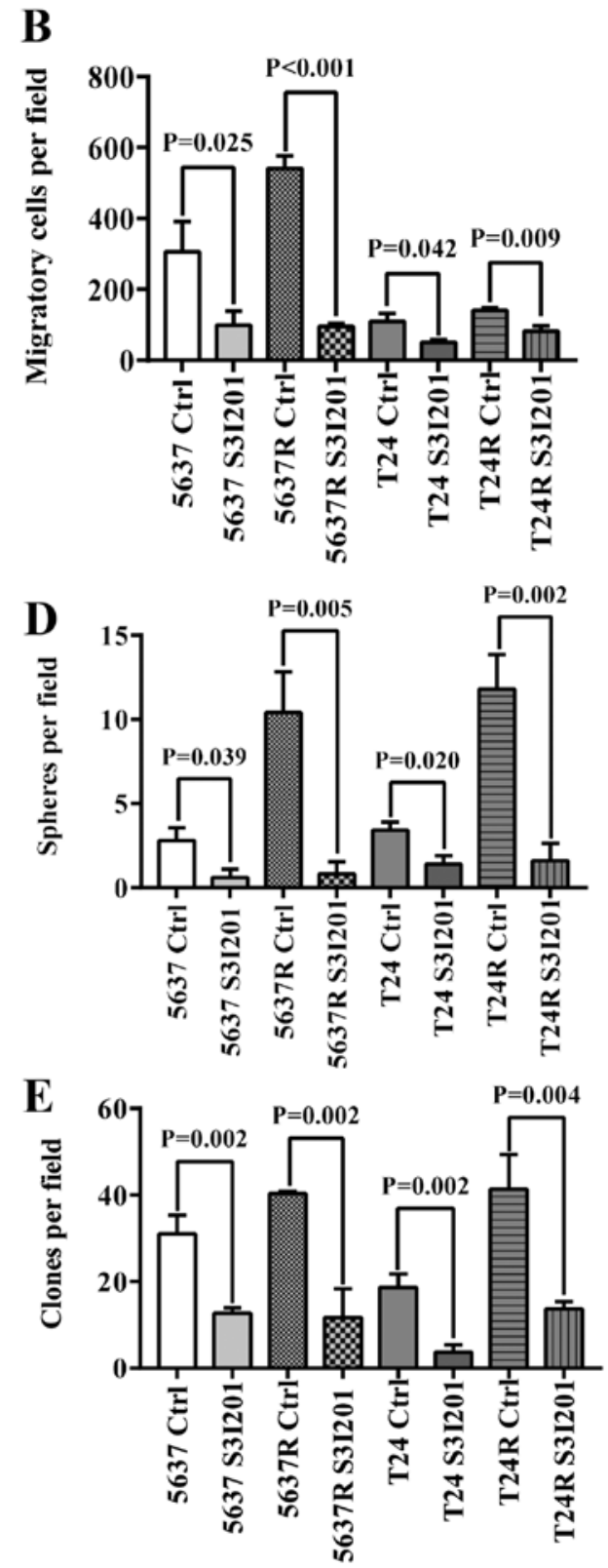

Figure 3. Elevated STAT3 phosphorylation in radio-resistant BCa cells is associated with an increase of tumor cell stemness. (A) Western blot analysis of pSTAT3 ${ }^{\text {Tyr705 }}$, pSTAT3 ${ }^{\text {Ser727 }}$, STAT3, MMP-2, MMP-9, KMT1A, GATA3 and JAK2, GAPDH served as a loading control. (B) Quantification of migration analysis of PBS-treated (Ctrl) and S3I201-treated BCa cells. (C) Quantification of invasion analysis of Ctrl and S3I201-treated BCa cells. (D) Quantification of tumorspheres formed by Ctrl and S3I201-treated BCa cells. S3I-201 was added to the culture to the final concentration of $50 \mu \mathrm{M}$. (E) Quantification of soft agar colony formation analysis of Ctrl and S3I201-treated BCa cells. The data are presented as the mean \pm SEM of triplicate experiments. STAT3, signal transduction and transcription activator 3; BCa, bladder cancer; p-, phosphorylated; MMP, matrix metalloproteinase; KMT1A, suppressor of variegation 3-9 homolog 1; GATA3, GATA binding protein 3; JAK2, Janus kinase 2; Ctrl, control; R, radio-resistant cells.

of KMT1A and JAK2 were upregulated, whereas GATA3 expression was decreased, followed by increased expression of MMP-2 and MMP-9 in both RR cell lines. Next, S3I-201 was used to inhibit STAT3 activation in BCa cells, which significantly decreased RR cell migration [5637 Ctrl vs. 5637 S3I-201, 305.8 \pm 85.21 vs. $99.4 \pm 39.52(\mathrm{P}=0.024) ; 5637 \mathrm{R}$ Ctrl vs. 5637R S3I-201, 540.8 \pm 36.41 vs. $95.0 \pm 9.38$ ( $\mathrm{P}<0.001)$; T24 Ctrl vs. T24 S3I-201, $109.8 \pm 23.40$ vs. $50.6 \pm 7.06(\mathrm{P}=0.042)$; and T24R Ctrl vs. T24R S3I-201, $141.8 \pm 6.65$ vs. $81.4 \pm 16.33$ ( $\mathrm{P}=0.009) ;$ Fig. 3B] and invasion [5637 Ctrl vs. $5637 \mathrm{~S} 3 \mathrm{I}-201$, $153.6 \pm 13.89$ vs. $35.4 \pm 7.50(\mathrm{P}<0.001)$; 5637R Ctrl vs. 5637R S3I-201, $495.0 \pm 68.12$ vs. $97.6 \pm 25.42(\mathrm{P}<0.001)$; T24 Ctrl vs. T24 S3I-201, $117.6 \pm 4.16$ vs. $77.6 \pm 11.22(\mathrm{P}=0.010)$; and $\mathrm{T} 24 \mathrm{R}$
Ctrl vs. T24R S3I-201, $147.2 \pm 9.28$ vs. $71.0 \pm 8.83(\mathrm{P}<0.001)$; Fig. 3C] compared with untreated control cells. Similarly, the tumorsphere-forming ability [5637 Ctrl vs. 5637 S3I-201, $2.8 \pm 0.75$ vs. $0.6 \pm 0.49(\mathrm{P}=0.040) ; 5637 \mathrm{R}$ Ctrl vs. $5637 \mathrm{R} \mathrm{S3I}-201$, $10.4 \pm 2.42$ vs. $0.8 \pm 0.75(\mathrm{P}=0.005)$; T24 Ctrl vs. T24 S3I-201, $3.4 \pm 0.49$ vs. $1.4 \pm 0.49(\mathrm{P}=0.020)$; and $\mathrm{T} 24 \mathrm{R} \mathrm{Ctrl}$ vs. T24R S3I-201, 11.8 \pm 2.04 vs. $1.6 \pm 1.02$ ( $\mathrm{P}=0.002)$; Fig. $3 \mathrm{D}]$ and soft agar colony-forming ability [5637 Ctrl vs. 5637 S3I-201, $31.0 \pm 4.32$ vs. $12.7 \pm 1.25(\mathrm{P}=0.002)$; $5637 \mathrm{R} \mathrm{Ctrl}$ vs. $5637 \mathrm{R}$ S3I-201, $40.3 \pm 0.47$ vs. $11.7 \pm 6.65$ ( $\mathrm{P}=0.002)$; T24 Ctrl vs. T24 S3I-201, $18.7 \pm 3.09$ vs. $3.7 \pm 1.70(\mathrm{P}=0.002)$; and $\mathrm{T} 24 \mathrm{R}$ Ctrl vs. T24R S3I-201, $41.3 \pm 8.06$ vs. $13.7 \pm 1.70(\mathrm{P}=0.004)$; Fig. $3 \mathrm{E}]$ of RR cells compared with those of the untreated control cells. 

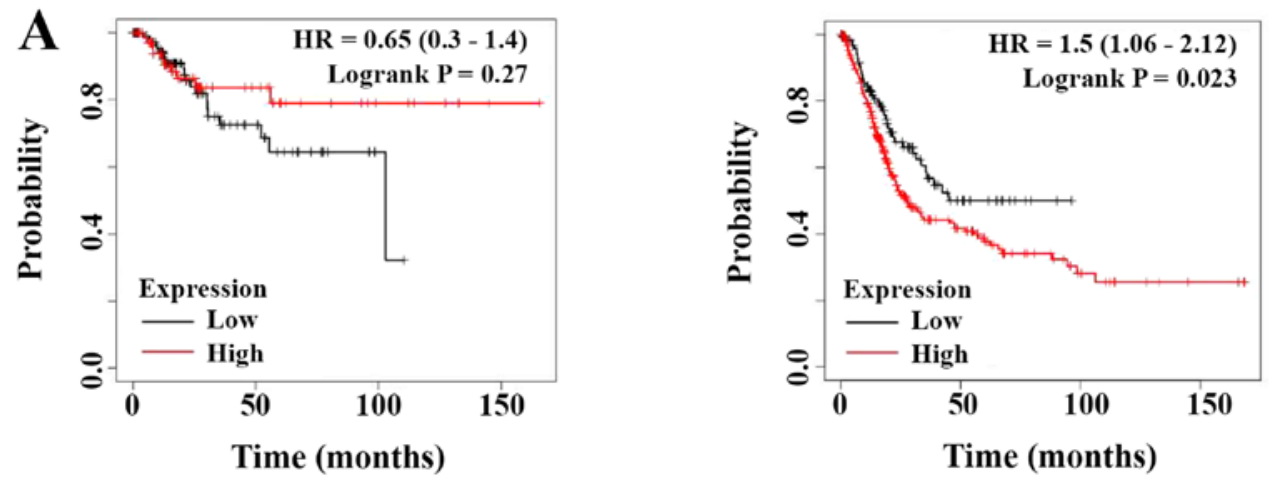

B
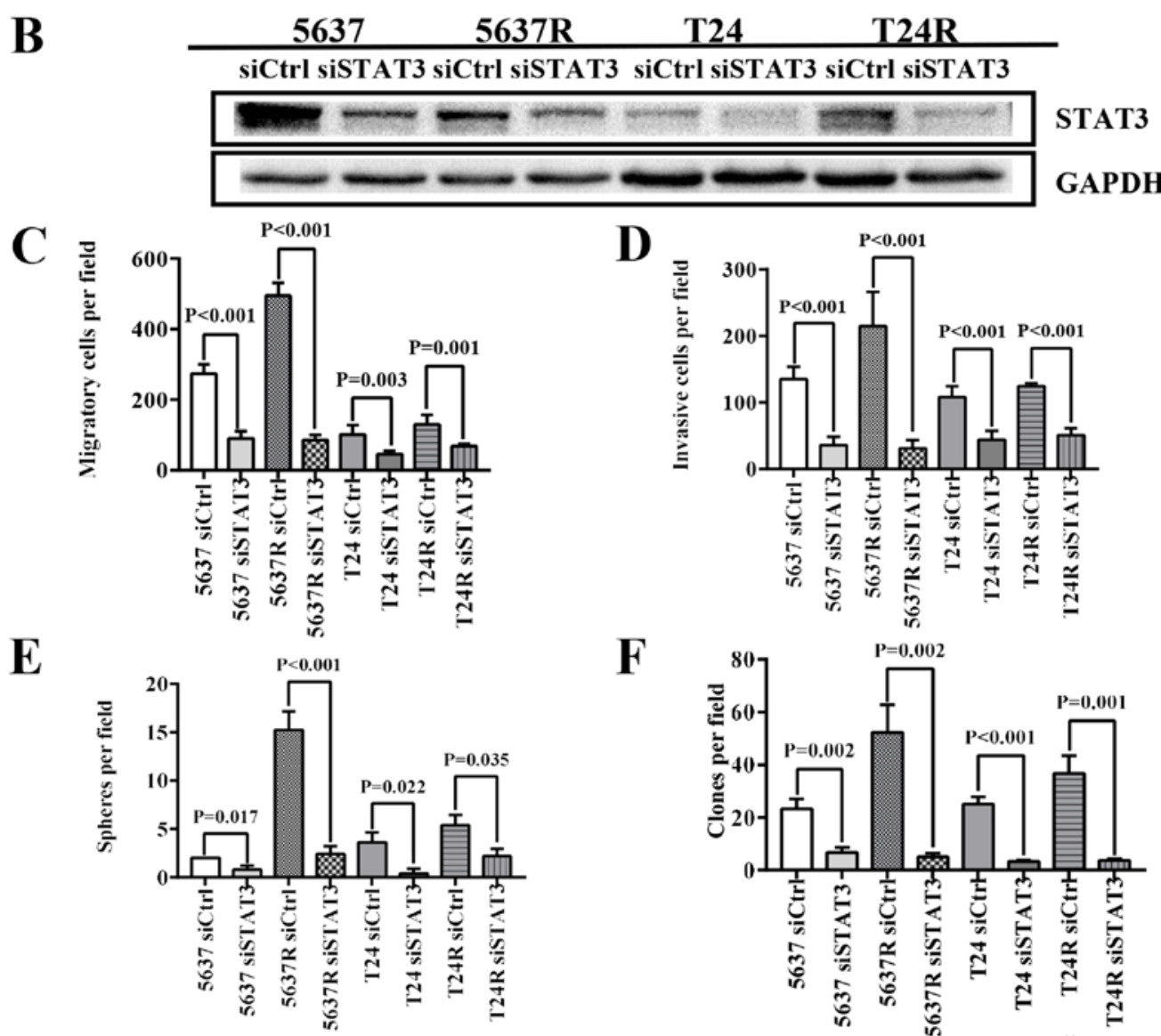

F
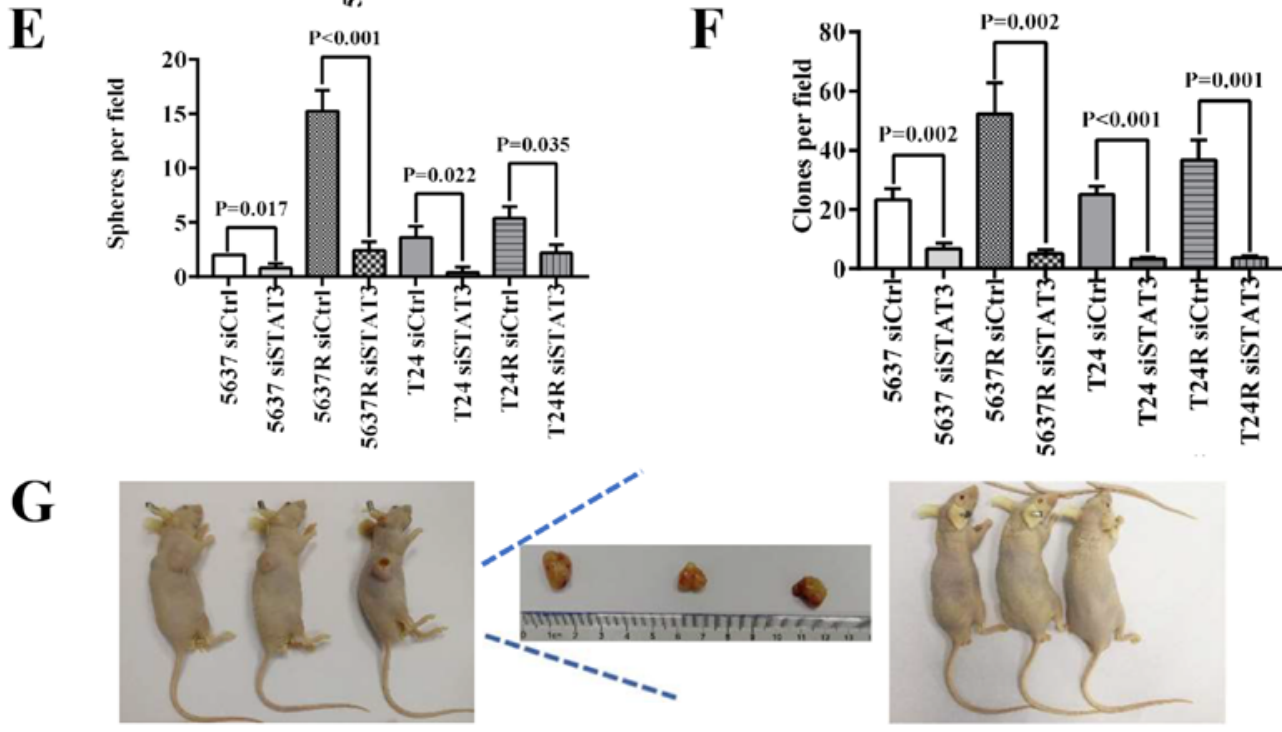

T24R siCtrl

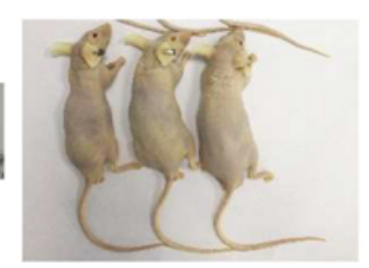

T24R siSTAT3

Figure 4. Depletion of STAT3 abrogates the self-renewal and tumorigenicity of human BCa cells. (A) Kaplan-Meier curves comparing the RFS and OS between patients with BCa expressing high or low levels of STAT3, a log-rank test was performed (left, RFS; right, OS). (B) Western blot analysis of STAT3, GAPDH served as a loading control. (C) Quantification of migration analysis of siCtrl and siSTAT3 BCa cells. (D) Quantification of invasion analysis of siCtrl and siSTAT3 BCa cells. (E) Quantification of tumorspheres formed by siCtrl and siSTAT3 BCa cells. (F) Quantification of soft agar colony formation analysis of siCtrl and siSTAT3 BCa cells. The data are presented as the mean \pm SEM of triplicate experiments. (G) Results of the tumor formation assays of siCtrl and siSTAT3 BCa cells $(\mathrm{n}=3)$. STAT3, signal transduction and transcription activator 3; BCa, bladder cancer; RFS, relapse-free survival; OS, overall survival; si-, small interfering RNA; Ctrl, control; HR, hazard ratio; R, radio-resistant cells.

STAT3 knockdown abrogates the self-renewal capacity and tumorigenicity of human $\mathrm{BCa}$ cells. In the following experiments, data from 405 patients with $\mathrm{BCa}$ were used to determine the relationship between STAT3 expression and patient prognosis using Kaplan-Meier plot and log rank test analysis. Although there were no significant differences in RFS between $\mathrm{STAT}^{\text {high }}$ and STAT3 ${ }^{\text {low }}$ patients $(\mathrm{HR}=0.65 ; \mathrm{P}=0.27$; 95\% CI, 0.3-1.4; Fig. 4A, left), decreased STAT3 mRNA levels 
were associated with higher $\mathrm{OS}$ in patients with $\mathrm{BCa}(\mathrm{HR}=1.5$; $\mathrm{P}=0.023$; 95\% CI, 1.06-2.12; Fig. 4A, right). STAT3 ${ }^{\text {high }}$ patients exhibited a shorter mean survival time (13.1 months) compared with STAT $3^{\text {low }}$ patients (18.83 months). Therefore, endogenous STAT3 expression was knocked down in BCa cells using the RNA interference method (Fig. 4B). Similar to cells treated with S3I-201, both parental BCa cells and their RR counterparts exhibited decreased motility and stemness, as indicated by the reduced migration [5637 siCtrl vs. 5637 siSTAT3, $273.8 \pm 26.11$ vs. $89.6 \pm 20.53$ ( $\mathrm{P}<0.001)$; 5637R siCtrl vs. 5637R siSTAT3, $495.0 \pm 35.92$ vs. $85.0 \pm 15.23(\mathrm{P}<0.001)$; $\mathrm{T} 24$ siCtrl vs. T24 siSTAT3, $101.2 \pm 27.26$ vs. $45.2 \pm 9.42(\mathrm{P}=0.003)$; and T24R siCtrl vs. T24R siSTAT3, $130.2 \pm 26.90$ vs. $67.8 \pm 7.09(\mathrm{P}=0.001)$; Fig. 4C] and invasion capabilities [5637 siCtrl vs. 5637 siSTAT3, $134.8 \pm 18.69$ vs. $36.2 \pm 12.60(\mathrm{P}<0.001) ; 5637 \mathrm{R}$ siCtrl vs. $5637 \mathrm{R}$ siSTAT3, $214.8 \pm 51.05$ vs. $31.4 \pm 12.40(\mathrm{P}<0.001)$; T24 siCtrl vs. T24 siSTAT3, $108.4 \pm 16.13$ vs. $44.4 \pm 12.93$ $(\mathrm{P}<0.001)$; and $\mathrm{T} 24 \mathrm{R}$ siCtrl vs. T24R siSTAT3, $124.6 \pm 4.51$ vs. $50.8 \pm 10.50(\mathrm{P}<0.001$; Fig. 4D] compared with the cells transfected with the siRNA negative control. The number of tumor spheres [5637 siCtrl vs. 5637 siSTAT3, $2.0 \pm 0.01$ vs. $0.80 \pm 0.40$ $(\mathrm{P}=0.017)$; $5637 \mathrm{R}$ siCtrl vs. 5637R siSTAT3, $15.2 \pm 1.94$ vs. $2.4 \pm 0.8(\mathrm{P}<0.001) ; \mathrm{T} 24$ siCtrl vs. T24 siSTAT3, 3.6 \pm 1.02 vs. $0.4 \pm 0.49(\mathrm{P}=0.022)$; and $\mathrm{T} 24 \mathrm{R}$ siCtrl vs. T24R siSTAT3, $5.4 \pm 1.02$ vs. $2.2 \pm 0.75(\mathrm{P}=0.035)$; Fig. $4 \mathrm{E}]$ and soft agar colonies [5637 siCtrl vs. 5637 siSTAT3, $23.3 \pm 3.68$ vs. $6.7 \pm 2.05$ $(\mathrm{P}=0.002) ; 5637 \mathrm{R}$ siCtrl vs. $5637 \mathrm{R}$ siSTAT3, $52.3 \pm 10.40$ vs. $5.0 \pm 1.41(\mathrm{P}=0.002) ; \mathrm{T} 24$ siCtrl vs. T24 siSTAT3, 25.0 \pm 2.83 vs. $3.3 \pm 0.47(\mathrm{P}<0.001)$; and $\mathrm{T} 24 \mathrm{R}$ siCtrl vs. T24R siSTAT3, $36.7 \pm 6.80$ vs. $3.7 \pm 0.47$ ( $\mathrm{P}=0.001)$; Fig. $4 \mathrm{~F}]$ was also lower in siSTAT3-treated BCa cells compared with their siCtrl counterparts. Moreover, T24R cells lost the ability to form tumors in immunodeficient mice following STAT3 suppression (Fig. 4G). These results indicated that the expression and activation of STAT3 play indispensable roles in the self-renewal maintenance and tumorigenicity of $\mathrm{BCa}$ cells. It has been reported that radiation may induce STAT3 phosphorylation via EGFR in lung cancer cells (28). Therefore, in the current study, the expression and activation level of EGFR was compared in both parent cells (5637 and T24) and their resistant counterparts (5637R and T24R). It was found that the mRNA expression of EGFR was lower in $5637 \mathrm{R}$ cells than that in 5637 cells, $1.0 \pm 0.01$ vs. $0.5 \pm 0.01$ (5637 vs. 5637R, $\mathrm{P}<0.001)$. However, compared with T24 cells, EGFR expression was notably increased in T24R cells, $1.0 \pm 0.02$ vs. $1.48 \pm 0.01$ (T24 vs. T24R, P<0.001) (Fig. S1). On the other hand, there was no significant difference in EGFR protein expression and phosphorylation between parent cells and resistant cells (Fig. S1).

\section{Discussion}

Unlike individuals with other types of cancer, the survival rate of patients with $\mathrm{BCa}$ has not improved over the past three decades (29). Due to its ability to preserve and promote the recovery of normal tissues, $\mathrm{FI}$ is widely used to treat $\mathrm{BCa}$. However, the redistribution of surviving tumor cells during the long-term FI period limits the efficacy of RT. In fact, redistributing tumors usually acquire ARR, which results in treatment failure (30). CSCs are unique subpopulations of cells within tumors. They are capable of self-renewal and differentiation, and possess high DNA damage repair abilities, reduced levels of reactive oxygen species production and low proliferative capacity (31). These functions impart resistance to a variety of therapeutic methods, including RT (32). Therefore, CSC-targeted drug screening presents a promising option for overcoming ARR.

Our previous study reported that FI increases STAT3 phosphorylation in BCa cells (18). STAT3 phosphorylation also suppressed the RR of glioblastoma stem cells, and was associated with improved patient prognosis (33). JAK2 is preferentially upregulated in colorectal CSC subpopulations, which is accompanied by the phosphorylation of STAT3. JAK2/STAT3 signaling plays a vital role in promoting tumor initiation and RR by limiting apoptosis and enhancing clonogenic potential (34). In 2017, the KMT1A-GATA3-STAT3 pathway was confirmed to be a novel signaling pathway for the self-renewal of human BCa stem cells (21). Consistent with the results of these reports, the results of the present study demonstrated that STAT3 is a key molecule linking RR, tumor invasiveness and cancer stem-like properties in BCa. $\mathrm{BCa}$ cells that survived FI were confirmed to be RR, possess increased migratory and invasive abilities, as well as enhanced CSC characteristics (Figs. 1 and 2), accompanied by increased phosphorylation levels of STAT3 (Fig. 3). Furthermore, knockdown of STAT3 expression and inhibiting its activation in BCa cells significantly inhibited the motility, and reduced the anchorage and proliferative abilities of tumor cells in low-adsorption media (Figs. 3 and 4). Notably, downregulating STAT3 expression also resulted in a loss of tumor formation ability in immunodeficient mice (Fig. 4G). Similarly, low STAT3 mRNA levels in patients with BCa was associated with improved OS, compared with STAT3 ${ }^{\text {high }}$ patients (Fig. 4A). Collectively, these data indicated that STAT3 plays an important role in the malignant progression, metastasis and recurrence of $\mathrm{BCa}$.

Compared with 5637 and T24 cells, a notable elevation in STAT3 phosphorylation at Tyr705 was observed in their RR counterparts (5637R and T24R cells). Possible upstream molecular mechanisms involving enhanced activation of STAT3 were also investigated; two stem cell-related signaling pathways (namely KMT1A-GATA3-STAT3 and JAK2-STAT3) were determined, and elevated expression of KMT1A and JAK2 were detected in RR cells, accompanied by the increased phosphorylation of STAT3 (Fig. 3B). In addition, the expression of MMP2 and MMP9, downstream effectors of STAT3 and key molecules in the regulation of tumor cell migration and invasiveness (35), were upregulated in both RR cell lines. When STAT3 phosphorylation was inhibited with S3I-201, the elevated expression of KMT1A, JAK2, MMP2 and MMP9 in RR cells was significantly suppressed, and the radiosensitivity of these cells was markedly enhanced (data not shown). Therefore, we hypothesized that the KMT1A-GATA3/JAK2-STAT3-MMP2/MMP9 signaling cascade is the key molecular mechanism involved in FI-induced tolerance to IR, enhancing the motility and CSC-like properties of BCa cells. However, the high cytotoxicity of S3I-201 limited its use in further animal experiments. The identification of novel inhibitors and appropriate methods of administration is still in progress. 
In the current study, only T24R cells formed tumors in nude mice. Although T24 cells (grade III) have a higher malignant capacity than 5637 cells (grade II), the latter were not able to form tumors under the current experimental conditions ( $1 \times 10^{6}$ cells/per mouse). However, compared with T24 cells, T24R cells exhibited an increased tumorsphere formation rate and formed more soft agar anchored clones, indicating that T24R cells possessed more potent self-renewal and proliferative abilities than T24 cells (Fig. 2). In addition, compared with $\mathrm{T} 24$ cells, T24R cells exhibited greater invasive capacity. It was therefore suggested that irradiation may enrich stem-like BCa cells and promote their ability to form tumors in vitro. When endogenous STAT3 expression was knocked down, T24R cells lost the ability to form tumors in vitro, further confirming that STAT3 plays an important role in maintaining the CSC-like phenotype of $\mathrm{BCa}$ cells.

At present, it is still unclear how IR induces STAT3 phosphorylation in BCa cells. Although EGFR activation in response to radiation has been demonstrated to be an upstream event of STAT3 phosphorylation (28), the present study found that only T24R cells showed increased mRNA expression of EGFR compared with T24 cells (Fig. S1). These results suggested that EGFR is not likely to be the key molecule linking IR and STAT3 phosphorylation. On the other hand, it was observed that JAK2/STAT3 activation was elevated in RR cells (Fig. 3), therefore it is speculated that IR-treated cells can release excessive inflammatory factors, such as IL-6, to activate the JAK-STAT signaling pathway. Our future studies will focus on elucidating the relationship among IR, cytokines and STAT3 activation following KMT1A and JAK2 overexpression.

In conclusion, the results of the present study demonstrated that FI induced RR, increased migration and invasion of cells, and enhanced the CSC-like characteristics of BCa cells, which were associated with the aberrant activation of STAT3. These findings suggested that STAT3 may be an effective therapeutic target to prevent the progression, metastasis and recurrence of $\mathrm{BCa}$ in patients receiving radiotherapy.

\section{Acknowledgements}

Not applicable.

\section{Funding}

The present study was supported by the National Nature Science Foundation of China (grant no. 31870846), the Natural Science Foundation of Shanghai (grant no. 18ZR1403600).

\section{Availability of data and materials}

The datasets used and/or analyzed during the current study are available from the corresponding author on reasonable request.

\section{Authors' contributions}

ZK designed the experiments. FW, XM and GM performed the experiments. FW, XM and XZ analyzed the data. ZK, FW and XM wrote the manuscript. All authors read and approved the final manuscript.

\section{Ethics approval and consent to participate}

All experiments were approved by the Committee for Ethical Use of Experimental Animals at Fudan University (approval no. 201802144S; Shanghai, China).

\section{Patient consent for publication}

Not applicable.

\section{Competing interests}

The authors declare that they have no competing interests.

\section{References}

1. Kaufman DS, Shipley WU and Feldman ASJL. Bladder Cancer. Lancet 374: 239-249, 2009.

2. Dong F, Xu T, Shen Y, Zhong S, Chen S, Ding Q and Shen Z: Dysregulation of miRNAs in bladder cancer: Altered expression with aberrant biogenesis procedure. Oncotarget 8: 27547-27568, 2017.

3. Park JC, Citrin DE, Agarwal PK and Apolo AB: Multimodal management of muscle-invasive bladder cancer. Curr Probl Cancer 38: 80-108, 2014.

4. Madersbacher S, Hochreiter W, Burkhard F, Thalmann GN, Danuser H, Markwalder R and Studer UE: Radical cystectomy for bladder cancer today - a homogeneous series without neoadjuvant therapy. J Clin Oncol 21: 690-696, 2003.

5. Smith AB, Jaeger B, Pinheiro LC, Edwards LJ, Tan HJ, Nielsen ME and Reeve BB: Impact of bladder cancer on health-related quality of life. BJU Int 121: 549-557, 2018.

6. Bajaj A, Martin B, Bhasin R, Hentz C, Block AM, Harkenrider MM and Solanki AA: The impact of academic facility type and case volume on survival in patients undergoing curative radiation therapy for muscle-invasive bladder cancer. Int J Radiat Oncol Biol Phys 100: 851-857, 2018.

7. Mitin T, Dengina N, Chernykh M, Usychkin S, Gladkov O, Degnin C, Chen Y, Nosov D, Tsimafeyeu I, Thomas CR Jr, et al: Management of muscle invasive bladder cancer with bladder preservation in Russia: A survey-based analysis of current practice and the impact of an educational workshop on clinical expertise. J Cancer Educ: Mar 4, 2020 (Epub ahead of print). doi: 10.1007/s13187-020-01728-y.

8. Shimura T: Acquired radioresistance of cancer and the AKT/GSK3 $3 /$ cyclin D1 overexpression cycle. J Radiat Res (Tokyo) 52: 539-544, 2011.

9. Sharda A, Rashid M, Shah SG, Sharma AK, Singh SR, Gera P, Chilkapati MK and Gupta S: Elevated HDAC activity and altered histone phospho-acetylation confer acquired radio-resistant phenotype to breast cancer cells. Clin Epigenetics 12: 4, 2020.

10. Anuja K, Kar M, Chowdhury AR, Shankar G, Padhi S, Roy S, Akhter Y, Rath AK and Banerjee B: Role of telomeric RAP1 in radiation sensitivity modulation and its interaction with CSC marker KLF4 in colorectal cancer. Int J Radiat Biol 96: 790-802, 2020.

11. Pajonk F, Vlashi E and McBride WH: Radiation resistance of cancer stem cells: The 4 R's of radiobiology revisited. Stem Cells 28: 639-648, 2010.

12. Vlashi E and Pajonk F: Cancer stem cells, cancer cell plasticity and radiation therapy. Semin Cancer Biol 31: 28-35, 2015.

13. Bighetti-Trevisan RL, Sousa LO, Castilho RM and Almeida LO: Cancer stem cells: Powerful targets to improve current anticancer therapeutics. Stem Cells Int 2019: 9618065, 2019.

14. Ishiguro T, Ohata H, Sato A, Yamawaki K, Enomoto T and Okamoto K: Tumor-derived spheroids: Relevance to cancer stem cells and clinical applications. Cancer Sci 108: 283-289, 2017.

15. Galoczova M, Coates P and Vojtesek B: STAT3, stem cells, cancer stem cells and p63. Cell Mol Biol Lett 23: 12, 2018.

16. Pattabiraman DR and Weinberg RA: Tackling the cancer stem cells - what challenges do they pose? Nat Rev Drug Discov 13: 497-512, 2014.

17. Lin L, Fuchs J, Li C, Olson V, Bekaii-Saab T and Lin J: STAT3 signaling pathway is necessary for cell survival and tumorsphere forming capacity in $\mathrm{ALDH}^{+} / \mathrm{CD} 133^{+}$stem cell-like human colon cancer cells. Biochem Biophys Res Commun 416: 246-251, 2011. 
18. Mao G, Yao Y and Kong Z: Long term exposure to $\gamma$ rays induces radioresistance and enhances the migration ability of bladder cancer cells. Mol Med Rep 18: 5834-5840, 2018.

19. Chang R, He H, Mao G and Kong Z: Upregulating DAB2IP expression via EGR-1 inhibition, a new approach for overcoming fractionated-irradiation-induced cross-tolerance to ionizing radiation and mitomycin $\mathrm{C}$ in tumor cells. Int J Radiat Biol 93: 386-393, 2017.

20. Sen N, Che X, Rajamani J, Zerboni L, Sung P, Ptacek J and Arvin AM: Signal transducer and activator of transcription 3 (STAT3) and survivin induction by varicella-zoster virus promote replication and skin pathogenesis. Proc Natl Acad Sci USA 109: 600-605, 2012.

21. Yang Z, He L, Lin K, Zhang Y, Deng A, Yong Liang Y, Li C, Wen T: The KMT1A-GATA3-STAT3 circuit is a novel self-renewal signaling of human bladder cancer stem cells. Clin Cancer Res 23: 6673-6685, 2017.

22. Nagy Á, Lánczky A, Menyhárt O and Győrffy B: Validation of miRNA prognostic power in hepatocellular carcinoma using expression data of independent datasets. Sci Rep 8: 9227, 2018.

23. Livak and Schmittgen: Analysis of relative gene expression data using real-time quantitative PCR and the 2-DDct method. Methods 25: 402-408, 2001.

24. Eun K, Ham SW and Kim H: Cancer stem cell heterogeneity: origin and new perspectives on CSC targeting. BMB Rep 50: $117-125,2017$.

25. Ahmad G and Amiji MM: Cancer stem cell-targeted therapeutics and delivery strategies. Expert Opin Drug Deliv 14: 997-1008, 2017.

26. Tran MN, Goodwin Jinesh G, McConkey DJ and Kamat AM: Bladder cancer stem cells. Curr Stem Cell Res Ther 5: 387-395, 2010.

27. Abubaker K, Luwor RB, Zhu H, McNally O, Quinn MA, Burns CJ, Thompson EW, Findlay JK and Ahmed N: Inhibition of the JAK2/STAT3 pathway in ovarian cancer results in the loss of cancer stem cell-like characteristics and a reduced tumor burden. BMC Cancer 14: 317, 2014.
28. Gao L, Li FS, Chen XH, Liu QW, FengJB, Liu QJ, Su X: Radiation induces phosphorylation of STAT3 in a dose- and time-dependent manner. Asian Pac J Cancer Prev 15: 6161-6164,201.

29. Berdik C: Unlocking bladder cancer. Nature 551: S34-S35, 2017.

30. Li JY, Li YY, Jin W, Yang Q, Shao ZM and Tian XS: ABT-737 reverses the acquired radioresistance of breast cancer cells by targeting Bcl-2 and Bcl-xL. J Exp Clin Cancer Res 31: 102, 2012.

31. Najafi M, Farhood B, Mortezaee K, Kharazinejad E, Majidpoor J and Ahadi R: Hypoxia in solid tumors: A key promoter of cancer stem cell (CSC) resistance. J Cancer Res Clin Oncol 146: 19-31, 2020.

32. Ohishi T, Koga F and Migita T: Bladder cancer stem-like cells: Their origin and therapeutic perspectives. Int J Mol Sci 17: 17, 2015.

33. Masliantsev K, Pinel B, Balbous A, Guichet PO, Tachon G, Milin S, Godet J, Duchesne M, Berger A, Petropoulos C, et al: Impact of STAT3 phosphorylation in glioblastoma stem cells radiosensitization and patient outcome. Oncotarget 9: 3968-3979, 2017.

34. Park SY, Lee CJ, Choi JH, Kim JH, Kim JW, Kim JY, Nam JS The JAK2/STAT3/CCND2 Axis promotes colorectal cancer stem cell persistence and radioresistance. J Exp Clin Cancer Res 38 399, 2019.

35. Huang S: Regulation of metastases by signal transducer and activator of transcription 3 signaling pathway: clinical implications. Clin Cancer Res 13: 1362-1366, 2007.

(i) (8) This work is licensed under a Creative Commons Attribution-NonCommercial-NoDerivatives 4.0 International (CC BY-NC-ND 4.0) License. 